\title{
Asynchronous Multi-Channel MAC for Vehicular Ad Hoc Networks
}

\author{
Chong Han, Mehrdad Dianati, Rahim Tafazolli, and Ralf Kernchen \\ Centre for Communications Systems Research (CCSR) \\ Department of Electronic Engineering, University of Surrey \\ Guildford, GU2 7XH, United Kingdom \\ Email: chong.han, m.dianati, r.tafazolli, r.kernchen@surrey.ac.uk
}

\begin{abstract}
This paper presents a new multi-channel MAC protocol for Vehicular Ad Hoc Networks, namely, Asynchronous Multi-Channel MAC (AMCMAC). The AMCMAC supports simultaneous transmissions on different service channels, as well as, allowing other nodes to make rendezvous with their provider/receiver or broadcast emergency messages on the control channel. We compare the performance of the proposed protocol with that of IEEE 1609.4 and Asynchronous Multi-channel Coordination Protocol (AMCP), in terms of throughput on control and service channels, channel utilization, and the penetration rate of successfully broadcast emergency messages. We demonstrate that AMCMAC outperforms IEEE 1609.4 and AMCP in terms of system throughput by increasing the utilization of control channel and service channels. In addition, AMCMAC mitigates both the multi-channel hidden terminal and missing receiver problems which occur in asynchronous multi-channel MAC protocols.
\end{abstract}

\section{INTRODUCTION}

Vehicular Ad hoc Networks (VANETs) [2] enable communications among nearby vehicles and between vehicles and nearby fixed infrastructures. These communication facilities are expected to be used for a variety of applications to improve safety of the future transport systems and provide many industrial and entertainment services. VANETs enable vehicle-to-vehicle (V2V) and vehicle-to-infrastructure (V2I) communications using wireless local area network (WLAN) technologies [1]-[4]. In particular, the IEEE $802.11 \mathrm{p}$ standard has been adopted to suit the highly dynamic propagation environment in VANETs and provide the QoS support for Intelligent Transport System (ITS) applications.

Recently, it has been reported that the original IEEE 802.11p standard based MAC sub-layer demonstrates a poor performance in dense VANETs, in provisioning QoS for different categories of applications ([5]-[7]). This seems to be a particularly important shortcoming for reliable deployment of safety related applications. Thus, multi-channel extension of the IEEE 802.11p standard, namely, the IEEE 1609.4 [8] has been proposed to help improve the service differentiation capability of the $802.11 \mathrm{p}$ standard [9] . In a multi-channel system, both non-safety and safety related applications could be provided on different channels. This could help improve QoS support for different application types by allocating them to different channels.

Although the IEEE 1609.4 standard helps provide a better QoS support, its MAC sub-layer suffers from a few shortcomings: 1) strict MAC sublayer synchronisation is required; 2) synchronous MAC sublayer operation results in inefficient utilisation of the control and service channels, i.e., utilisation cannot inherently exceed 50\%; 3) fixed duration of control and service time intervals prohibits intelligent allocation of time intervals in response to variable traffic demands; and 4) there is possibility of contention in service channel that may result in inefficiency due to possible collisions. Motivated by the inherent problems of IEEE 1609.4, some enhancements ([12]-[15]) and alternative solutions ([16]-[22]) have been proposed in recent years.

The two categories [10] of multi-channel MAC protocols, namely Common Hopping ([16], [17]) and Parallel Rendezvous protocols ([18], [19]), either requires tight clock synchronization or requires the source node to know the hopping sequence of the destination node which increases the signalling overhead. In addition, the hopping time penalty is not negligible in both categories of protocols, since the time of switching channels is comparable with the time consumed on transmitting an RTS packet [10].

Asynchronous multi-channel MAC protocols ([20]-[22]) are also proposed in literature. This category of multi-channel protocols usually requires no strict time synchronization and allows nodes to hop among channels independently to make the best use of channel resource. The Asynchronous Multi-channel Coordination Protocol (AMCP) [22] uses a dedicated control channel as in IEEE 1609.4 and requires no time synchronization among nodes to operate on data channels. However, the channel selection negotiation proposed in [22] may cause a second round negotiation, which results in extra overhead. In addition, [22] tries to solve the multi-channel hidden terminal problem by recoding all other service channels busy for a duration, which reduces the channel utilization of service channels and the probability of making successful channel negotiations in the next negotiation. In [20], the authors introduce cooperation among neighbours into multi-channel MAC to help to choose available service channels. However, as concluded in their paper, node mobility will affect the accuracy of the (one- and two-hop) neighbour information. In addition, due to the multiple phases in the handshake process, the protocol is not suitable for vehicular networks in which node mobility may be dramatic. 
This paper proposes a novel straightforward asynchronous multi-channel MAC protocol, namely Asynchronous Multi-Channel MAC (AMCMAC), that supports multiple transmissions simultaneously on different service channels. AMCMAC relies on only one single radio which is suitable for practical systems. Comparing to AMCP, the proposed scheme adopts different channel negotiation process, different methods to tackle multi-channel hidden terminal and missing receiver problems, load balancing channel selection mechanism and different post-transmission process. According to [11], which presents a comparative study of well known multi-channel MAC protocols, among the three multi-channel MAC schemes, AMCP is the most suitable asynchronous multi-channel MAC scheme for benchmarking in vehicular networks. Hence, we choose the representative work $\mathrm{AMCP}$ as one of the benchmarks in our paper. We compare the system performance of the AMCMAC scheme with that of the IEEE 1609.4 and AMCP in this paper. Simulation results demonstrate that the AMCMAC outperforms both of the aforementioned schemes in terms of system throughput on both control and service channels. AMCMAC achieves higher channel utilization on all channels, and takes load balancing on service channels into account.

The rest of the paper is organized as follows. Section II reviews the work of the IEEE 1609.4 and AMCP. In Section III, the AMCMAC is proposed. Section IV presents the simulation results and analysis. Finally, conclusion is given in Section V.

\section{BACKGROUND}

In this section, the relevant aspects of the IEEE 1609.4 and AMCP are illustrated. Then, several protocol design issues and challenges for asynchronous multi-channel MAC protocols are discussed.

\section{A. IEEE 1609.4}

IEEE 1609.4 is the standard of multi-channel operation for VANETs. In IEEE 1609.4, as shown in Fig. 1, the channel access time is divided into sync intervals. Each interval contains guard intervals and alternating fixed-length intervals called $\mathrm{CCH}$ interval and $\mathrm{SCH}$ interval. During the $\mathrm{CCH}$ interval, all nodes monitor the $\mathrm{CCH}$ for exchanging safety messages and other control packets. During the SCH interval, nodes exchange the non-safety application data on SCHs.

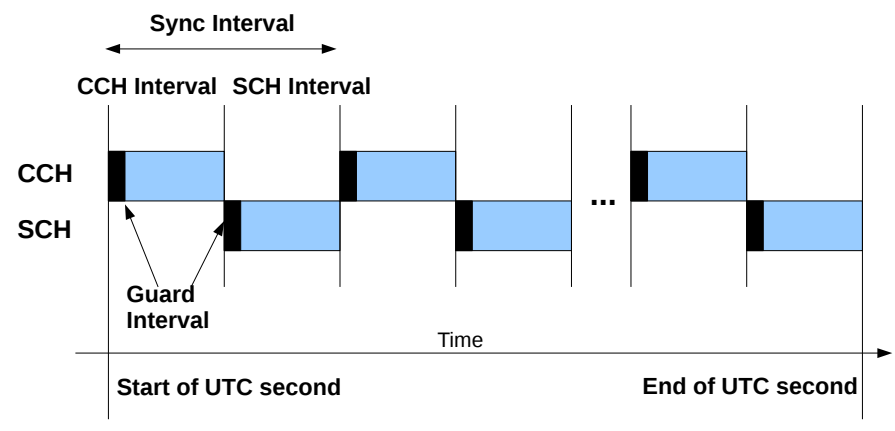

Fig. 1. Sync interval and its components in IEEE 1609.4.

The current version of the IEEE 1609.4 MAC does not provide a high level of QoS guarantee and strict differentiating of services for the potential real time applications in the dense network scenarios. First, the duration of CCH and SCH intervals are fixed in the standard IEEE 1609.4. In a congested urban areas with dense VANETs, large volume of safety and control messages may need to be delivered in the $\mathrm{CCH}$ interval. The fixed length of $\mathrm{CCH}$ may not be able to provide sufficient bandwidth in these scenarios. This may affect the utilization of service channels as the nodes may not be able to perform negotiations on a highly congested CCH. On the other hand, if the node density is sparse, the CCH interval will may be left idle for significant periods of time.

In addition, since nodes do not specifically determine the duration of service channel use during the negotiation process on the control channel, there will be possibilities of collisions on the service channels.

\section{B. $A M C P$}

The AMCP is an asynchronous multi-channel coordination protocol which does not require synchronous hopping from service channels to the control channel and vice versa. Each node in AMCP maintains a channel table with $\mathrm{N}$ entries corresponding to $\mathrm{N}$ data channels. In the channel negotiation process of $\mathrm{AMCP}$, the sender chooses a preferred channel and sends the information within RTS; if the channel is available for the receiver, a Confirming CTS is sent back. After receiving the Confirming CTS, both nodes switch to the data channel they agree with to start the transmission. However, if the channel chosen by the sender is unavailable for the receiver, a Rejecting CTS containing index 0 and a list of available channels of the receiver will be sent to the sender. Then if one of the available channels in the list is also available for the sender, it retransmits an RTS inserting the new channel. This process may result in a second round of channel negotiation when the preferred channel of the sender is not available for the receiver. This will result in inefficient channel utilization in AMCP. 
In addition, nodes may mark many service channels busy for unnecessarily long periods of time in their local tables. If a node does not have a precise status of a specific data channel, it sets the channel unavailable to avoid possible collisions on that channel. This helps to reduce the probability of collisions on data channels; however it also causes other problems: 1 ) it may unnecessarily waste the opportunities for a node to make successful channel negotiations; 2) nodes may always use a specific channel it does not obtain enough information about the status of the other channels.

Besides the multi-channel hidden terminal and missing receiver problems, there is another common problem for asynchronous multi-channel MAC protocols. In asynchronous multi-channel MAC protocols, when a node attempts to broadcast an emergency message, it broadcasts the packet on the CCH without the RTS/CTS handshake. However, it is important to note that emergency messages will not be received by the nodes that are involved in an ongoing communication on the service channels. It has to be mentioned that in asynchronous multi-channel MAC protocols, the problem of missing emergency messages cannot be completely mitigated. One possible solution can be rebroadcast/forwarding of emergency messages by the nodes which have received the message. Since emergency messages are usually forwarded/disseminated to the neighbours or nodes within a relevant area, the concern of missing emergency messages should not be severe.

In summary, the aforementioned multi-channel MAC protocols suffer from some shortcomings that we discussed in this section. Thus, we propose a new asynchronous multi-channel MAC scheme in the following section taking into account the requirements for potential VANET applications.

\section{Asynchronous Multi-Channel MAC (AMCMAC)}

Considering the strength and weakness in the existing solutions as well as the specific features of VANETs, an asynchronous multi-channel MAC is proposed. In this section, first, the overview of the proposed protocol is provided. Then, the detailed design of the proposed scheme is discussed. Finally, we explain how the multi-channel coordination problems are addressed.

AMCMAC protocol aims to overcome the weaknesses in existing multi-channel protocols such as time synchronization difficulty, hopping time penalty, missing receiver problem, and multi-channel hidden terminal problem. Meantime, the aim is to improve the performance of multi-channel operation.

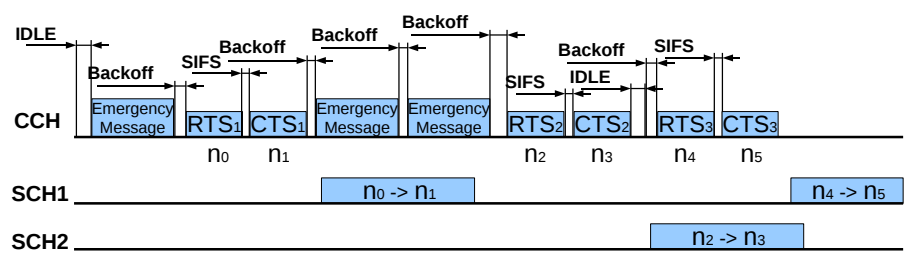

Fig. 2. Proposed asynchronous multi-channel MAC scheme.

Fig. 2 shows the basic channel access mechanism of the proposed MAC protocol. All the nodes, which are not using SCHs to transmit data stay with $\mathrm{CCH}$, and listen to the $\mathrm{CCH}$ if they do not have packets to transmit in their queues. Each node keeps a local SCHs entry table, which records the status of each channel and the time when the channel will be freed, based on the information the node collects by listening to $\mathrm{CCH}$. An example of the local SCHs entry table is shown in Table I. For

TABLE I

AN EXAMPLE OF THE LOCAL SCHS ENTRY TABLE

\begin{tabular}{|c|c|c|}
\hline channel & status[channel] & free_time[channel] \\
\hline 1 & 1 & $1.234837234 \mathrm{~s}$ \\
\hline 2 & 0 & 0 \\
\hline 3 & 0 & 0 \\
\hline 4 & 1 & $1.235023677 \mathrm{~s}$ \\
\hline 5 & 1 & $1.233102348 \mathrm{~s}$ \\
\hline 6 & 0 & 0 \\
\hline
\end{tabular}

instance, according to this local SCHs entry table, the node deems that $\mathrm{SCH} 2$, SCH3 and SCH6 are idle at the moment, while SCH1 is busy until $1.234837234 \mathrm{~s}$.

Once a node needs to broadcast/unicast a packet, it competes with other stations for the access of the CCH. If the channel is sensed idle, after a backoff time the sender broadcasts an RTS packet through $\mathrm{CCH}$. Inside the RTS packet, a list of available $\mathrm{SCH}$ for the sender is added. The destination node obtains the list from the RTS and checks its local SCHs entry table. If the destination finds an available channel which also is available for the sender, it replies a CTS after a period of SIFS with 
the channel information that they will use for the transmission. After a successful handshake, the two nodes will switch to the $\mathrm{SCH}$ they agreed to finish the data transmission. Transmissions can simultaneously proceed on different service channels.

More specific design aspects of the proposed scheme are given in the following subsections.

\section{A. Time Synchronization}

To avoid the synchronization problem that exists in most split phase protocols (e.g., [8]-[14]), an asynchronous multi-channel MAC scheme is designed. The nodes without packets in queues stay on the $\mathrm{CCH}$, listening to the broadcast emergency messages and channel negotiation information. Unlike the IEEE 1609.4 [8], nodes do not have to switch among CCH and SCHs every $50 \mathrm{~ms}$, hence not only tight time synchronization is not needed, but also the service channels can be utilized all the time. Thus, the utilization of SCHs and $\mathrm{CCHs}$ are increased, which brings performance gain in terms of throughput.

\section{B. Channel Negotiation and Selection Strategy}

Taking the weaknesses of channel negotiation process in AMCP into account, the proposed AMCMAC adopts different channel negotiation mechanism and different channel selection strategy, aiming to offer more efficient channel negotiations and load balancing.

First, as indicated in the fourth paragraph in this section, the decision of which $\mathrm{SCH}$ to be used is decided by the destination node; hence, no more renegotiation is needed. While in AMCP, if the "prefer" SCH is not available for the destination node, a second round of negotiation will be initiated by the destination node. Due to the fast movement of the nodes in VANETs, the shorter a handshake takes, the higher probability of successful transmission will be achieved.

Secondly, in a multi-channel MAC, in some occasions, all the SCHs may seem unavailable for a sender/receiver. On the sender side, it will not send any packet except emergency messages, since it is not possible to make an agreement with other nodes. The sender restarts a backoff procedure to try to access the $\mathrm{CCH}$ later if it has packets to transmit. While if all the available SCHs are not available for the receiver, it means that the pair of nodes cannot make any agreement on SCH selection. Hence, on the receiver side, we make the destination node drop the received RTS packet directly, instead of replying a CTS. This mechanism reduces the time wasted on transmitting a CTS with failure information, hence other nodes may access to the $\mathrm{CCH}$ to make possible channel negotiation or to broadcast emergency messages.

In addition, each node updates its local SCHs entry table by listening to the $\mathrm{CCH}$. Once a node becomes a destination, it chooses the least used SCH in order to balance the load on service channels. Considering such scenarios, one of the SCHs is heavily used while other SCHs are seldom chosen for transmissions; load balancing is needed, since not only a higher probability of collisions may occur on the heavy loaded $\mathrm{SCH}$, but also it may become the bottleneck of the network.

\section{Multi-channel Hidden Terminal Problem}

For a multi-channel MAC, hidden terminal problem is different than that of single-channel MAC. As Fig. 3 illustrates, the multi-channel hidden terminal problem occurs in the following situation. For example, consider a scenario where pair $(2,3)$

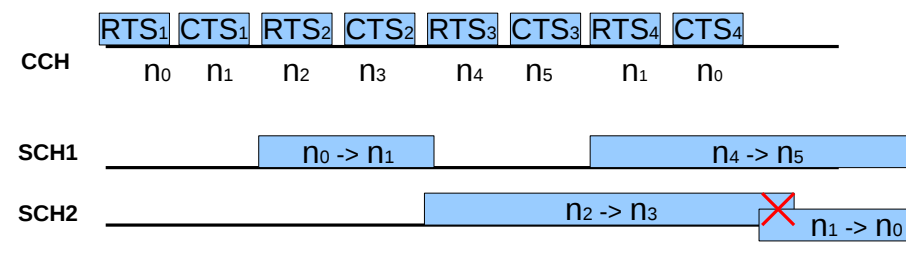

Fig. 3. Multi-channel Hidden Terminal Problem.

are involved in an ongoing communication on SCH2. If pair $(0,1)$ misses the corresponding RTS/CTS between pair $(2,3)$, they may choose the same service channel. This is known as the multi-channel hidden terminal problem. This problem causes collisions on SCHs and degrades the network performance.

The AMCMAC aims to solve this problem: when nodes switch to an $\mathrm{SCH}$, first, they listen to the service channel for a short period of time before transmission. This duration can vary according to the requirements of the system, such as the guard interval after frequency switching and channel condition in specific scenarios. However, it must be larger than $32 \mathrm{~ms}$ which is the duration of an SIFS. During the carrier sensing, if the channel is not idle, i.e., another pair is currently using that particular channel, the nodes record that SCH as busy for a certain duration (e.g., a duration of transmissions of DATA and ACK) and return to the $\mathrm{CCH}$ to renegotiate for another available $\mathrm{SCH}$.

In single hop networks, this measure helps to mitigate multi-channel hidden terminal problem. In multi-hop networks, assuming that one of the nodes fails to sense the current SCH's busy status, and remains on the service channel, while its 
sender/receiver has left the SCH. If the destination node remains on $\mathrm{SCH}$, once it receives any packet from other pair of nodes, the node knows the SCH is occupied by other nodes and returns to $\mathrm{CCH}$. If the remaining node is the sender node, it must be outside the communication range of the transmitting node of the other pair of nodes. If the receiving node of the other pair of nodes is also outside its communication range, there will be no interference even though the sender node without partner transmits a packet on $\mathrm{SCH}$.

However, in the worst case, if the node acts like a hidden terminal on the service channel to the other pair of nodes, either employing RTS/CTS mechanism on SCHs or introducing cooperation among nodes on $\mathrm{CCH}$ can help to mitigate the problem. This work will be continued in our future work.

\section{Missing Receiver Problem}

Another problem in asynchronous multi-channel protocols is called missing receiver problem, which occurs if the destination node is absent in a channel negotiation due to participating an ongoing transmission on another service channel, or unavailable in the network. This problem also contributes to inefficient channel utilization as a node may have to wait for a long time to confirm an unsuccessful handshake as in IEEE 802.11a, e, p and IEEE 1609.4. Nodes have to set their NAV timers for duration of CTS packet plus an SIFS. Before the NAV timer expires, all the nodes except the destination node wait for the response, i.e., the CTS packet. If the destination node is not available any more or currently busy on other SCHs, the other nodes will have to stay in a waiting mode for a long time.

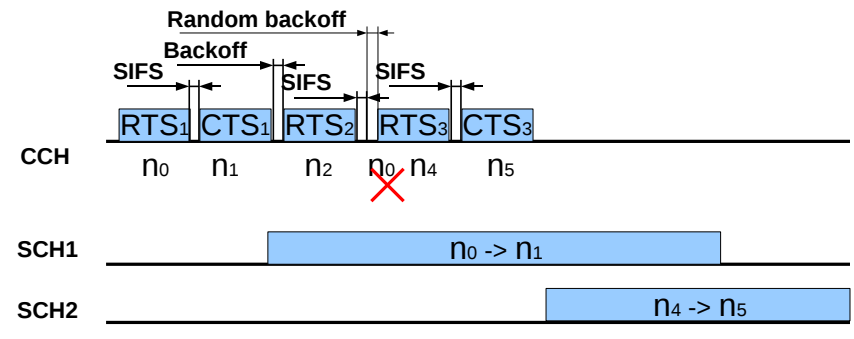

Fig. 4. Missing Receiver Problem.

The proposed solution for missing receiver problem is shown in Fig. 4. A shorter NAV timer is adopted to reduce the channel access time due to the unsuccessful handshakes. Once receiving an RTS packet, nodes on CCH (excluding the sender and destination nodes) set a timeout period, Timeout $_{C T S}$, given by (1).

$$
\text { Timeout }_{C T S}=\delta+S I F S+\delta+(\text { Node ID) } \bmod 31,
$$

where $\delta$ is the propagation delay. After this short timeout, if the receiver does not send a CTS message, the other nodes could start their own backoff procedures, immediately. A random duration, the 4th term in the right side of (1), is introduced to avoid RTS collisions on the $\mathrm{CCH}$.

\section{Performance Evaluation}

We evaluate AMCMAC using the well-known simulation tool, NS-2 [23], from Lawrence Berkeley National Laboratory. The simulation scenario considers a single hop reference area that comprise a $500 \mathrm{~m} \times 500 \mathrm{~m}$ urban area, where vehicles are randomly distributed and the speed range is from $2 \mathrm{~m} / \mathrm{s}$ to $15 \mathrm{~m} / \mathrm{s}$. The density of vehicles in the area varies by changing the number of nodes, from 10 to 100 in the simulations. To evaluate the performance of the multi-channel approach, first, a scenario without emergency messages (AC0) is considered. In the "Non-AC0" scenario, each vehicle has three categories of traffic (i.e., $\mathrm{AC} 1, \mathrm{AC} 2$ and $\mathrm{AC} 3$ ) which represent packets for non-safety related applications. There is always a packet in each AC queue. We also consider another scenario with emergency messages, in a situation where AC1, AC2 and AC3 flows are saturated and the packet arrival interval of AC0 flow is 1s. The frequency of AC0 flow is configured at a low level since emergency messages do not frequently occur in the network. The rest of the major simulation parameters are chosen from the IEEE 1609.4 standard as listed in Table. II.

In the first set of simulations, the data rate for $\mathrm{CCH}$ is set to $12 \mathrm{Mbps}$, and $6 \mathrm{Mbps}$ for each $\mathrm{SCH}$. First, the average network throughput per service channel in "Non-AC0" scenarios is measured in IEEE 1609.4, AMCP and AMCMAC, shown in Fig. 5. In this case, AMCMAC outperforms IEEE 1609.4 and AMCP. AMCMAC and AMCP outperform IEEE 1609.4 in terms of system throughput for different number of nodes in the reference area. AMCMAC obtains about $43 \%$ throughput per service channel on average, which is about $15 \%$ above the throughput obtained in AMCP. While in the scenario employing IEEE 1609.4, the average throughput per service channel is increased linearly due to the small amount of transmissions in each SCH interval. Within each 50ms SCH interval, there are a few transmissions to be completed, thus service channels are 
TABLE II

PARAMETER SETTING

\begin{tabular}{|l|l|}
\hline Packet payload & 1024 bytes \\
\hline Slot time & $13 \mu \mathrm{s}$ \\
\hline SIFS & $32 \mu \mathrm{s}$ \\
\hline DIFS & $58 \mu \mathrm{s}$ \\
\hline$\delta$ & $2 \mu \mathrm{s}$ \\
\hline DataRate for SCHs & $6 \mathrm{Mbps}$ \\
\hline BasicRate for CCH & $3,12,27 \mathrm{Mbps}$ \\
\hline AIFS[0] & 2 \\
\hline AIFS[1] & 3 \\
\hline AIFS[2] & 6 \\
\hline AIFS[3] & 9 \\
\hline AC0 $C W_{\min }$ & 3 time slots \\
\hline AC0 $C W_{\max }$ & 7 time slots \\
\hline AC1 $C W_{\min }$ & 3 time slots \\
\hline AC1 $C W_{\max }$ & 15 time slots \\
\hline AC2 $C W_{\min }$ & 7 time slots \\
\hline AC2 $C W_{\max }$ & 1023 time slots \\
\hline AC3 $C W_{\min }$ & 15 time slots \\
\hline AC3 $C W_{\max }$ & 1023 time slots \\
\hline
\end{tabular}

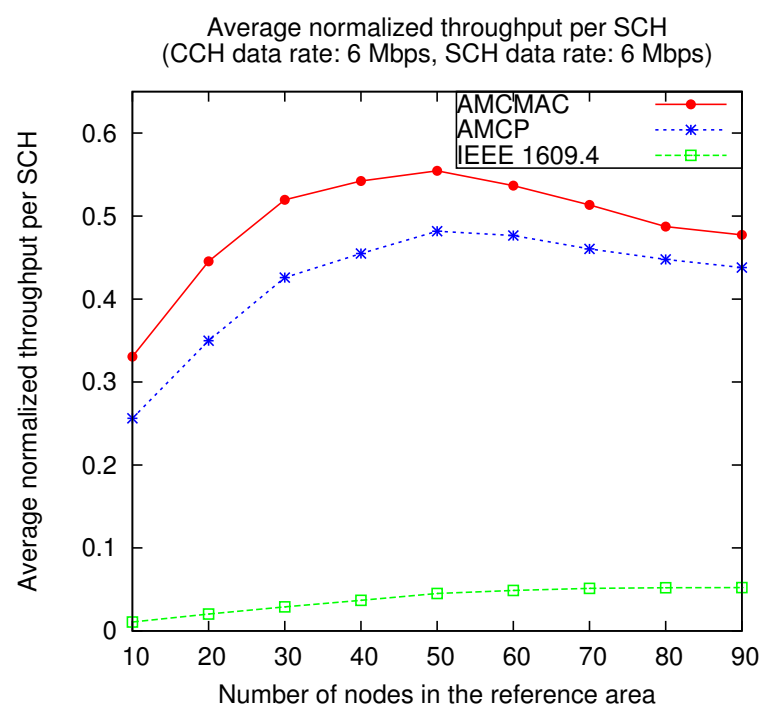

Fig. 5. Average normalized throughput per SCH as the number of stations varies.

not fully utilized. Hence, the throughput on service channel of IEEE 1609.4 is much lower than the other two asynchronous multi-channel MAC proposals.

Now we analyze the channel utilization of control channel in these three MAC protocols. In Fig. 6, we calculate the time when each channel is in busy status, i.e., the channel busy percentage for the $\mathrm{CCH}$ and average value for service channels in IEEE 1609.4, AMCP and AMCMAC. From this figure, the CCH is actually busy for $40 \%-45 \%$ of the time in AMCMAC and AMCP. Taking the contention period (i.e., AIFS + backoff timer) into account, the utilization of CCH in both systems is quite high. However, the throughput of $\mathrm{CCH}$ is quite low as shown in Fig. 7. The low throughput of $\mathrm{CCH}$ is partially due to the collisions on control channel, and partially due to the limitation of the transmission of physical layer convergence procedure (PLCP) preambles. The PLCP preamble, which consists of 10 short symbols and two long symbols, is used for synchronization. The PLCP preamble is transmitted at the data rate of 3 Mbps (defined in the IEEE 1609.4). Hence, although the control channel is utilized most of the time, the throughput of control channel is not high. To achieve higher throughput and more successful channel negotiations per unit time, the data rate for PLCP preamble can be changed to high value if there 


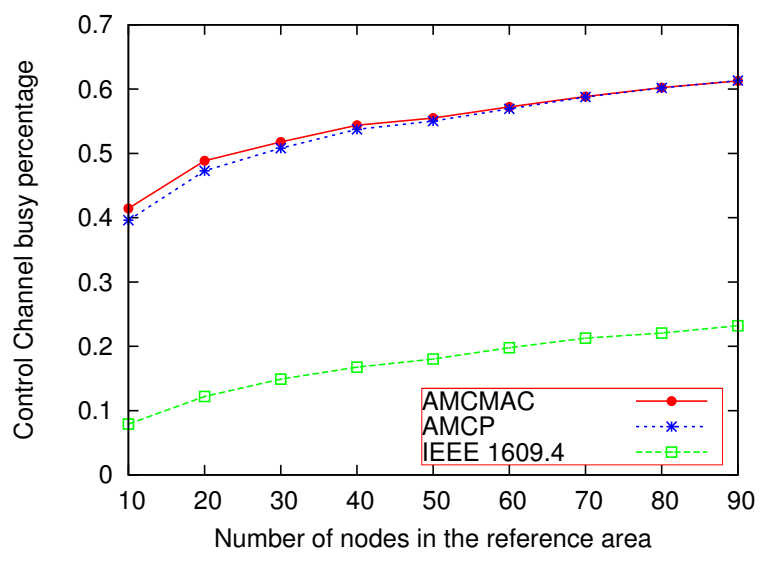

Fig. 6. $\mathrm{CCH}$ channel busy percentage.

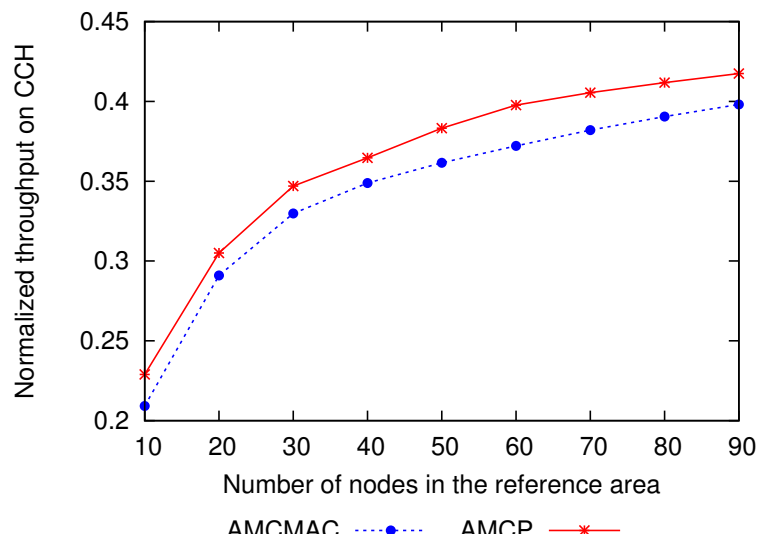

Fig. 7. Overall system throughput as the number of stations varies.

is no other reason to transmit the PLCP preambles in a low data rate. Furthermore, from Fig. 7 the throughput on $\mathrm{CCH}$ in low density scenarios in AMCP is higher than that in AMCMAC. However, due to the unnecessary round of channel selection negotiation in AMCP, the higher throughput on $\mathrm{CCH}$ offers fewer successful negotiations; hence the average throughput on service channels is not higher than that in AMCMAC.

Finally, as mentioned in Section I, there is a common problem for asynchronous multi-channel MAC protocols that emergency messages can not be received by all other nodes, since some nodes may be busy transmitting data on service channels. Another set of simulations compares the penetration rate of emergency messages in terms of the percentage of the nodes that successfully receive a non-collided emergency message. From the simulation results, the penetration rate of successfully broadcast emergency messages in the proposed protocol is over $94 \%$ in scenarios with both asynchronous multi-channel MACs. For high node density, the penetration rate of successfully broadcast emergency messages is almost as good as the IEEE 1609.4. Hence, although there are some nodes that miss emergency messages due to the absence on $\mathrm{CCH}$, the emergency messages are disseminated to most of the vehicles. If rebroadcast/forwarding is taken into account, the reception of emergency messages can be as good as synchronous multi-channel MAC protocols.

\section{CONCLUSION}

This paper proposes an Asynchronous Multi-Channel MAC (AMCMAC) protocol. The results of our performance analysis demonstrate that the proposed scheme outperform the standard IEEE 1609.4 and another asynchronous multi-channel scheme AMCP in terms of system throughput on both service channel and control channel, channel utilization and the penetration rate of successfully broadcast emergency messages. In addition, the proposed scheme mitigates two important issues, namely, the multi-channel hidden terminal problem and the missing receiver problem, in asynchronous multi-channel MAC operation. 


\section{ACKNOWLEDGMENT}

The work was done within the joint research project DRIVE C2X, which is funded by DG Infso of the European Commission within the 7th Framework Program.

\section{REFERENCES}

[1] H. Hartenstein and K. Laberteaux, "A tutorial survey on vehicular ad hoc networks," IEEE Commun. Mag., vol. 46, no. 6, pp. 164-171, Jun. 2008.

[2] Y. Toor, P. Muhlethaler and A. Laouiti, "Vehicle Ad Hoc networks: applications and related technical issues," IEEE Commun Surv Tutorials, vol. 10, no. 3, pp. 74-88, 2008

[3] T. Kosch, C. J. Adler, S. Eichler, C. Schroth and M. Strassberger, "The scalability problem of vehicular ad hoc networks and how to solve it," IEEE Commun. Mag., vol. 13, no. 5, pp. 22-28, Oct. 2006.

[4] H. Menouar, F. Filali and M. Lenardi, "A Survey and Qualitative Analysis of MAC Protocols for Vehicular Ad Hoc Networks," IEEE Trans. Wireless Commun., vol.13, pp.30-35, 2006.

[5] C. Han, M. Dianati, R. Tafazolli and R. Kernchen, "Throughput Analysis of the IEEE 802.11p Enhanced Distributed Channel Access Function in Vehicular Environment,"2010 IEEE 72nd Vehicular Technology Conference Fall (VTC 2010-Fall), Sep. 2010, pp. 1-5.

[6] S. Eichler, "Performance Evaluation of the IEEE 802.11p WAVE Communication Standard," in Proc. 2007 IEEE 66th Vehicular Technology Conference (VTC-2007 Fall),Sep. 2007, pp. 2199-2203.

[7] J. W. Robinson and T. S. Randhawa, "Saturation throughput analysis of IEEE 802.11e enhanced distributed coordination function," IEEE J. Sel. Areas Commun., vol. 22, no. 5, Jun. 2004, pp. 917-928.

[8] IEEE Trial-Use Standard for Wireless Access in Vehicular Environments (WAVE) - Multi-Channel Operation, IEEE Std 1609.4-2006, pp. c1 -74, 2006.

[9] Wireless LAN Medium Access Control (MAC) and Physical Layer (PHY) specifications Amendment 7: Wireless Access in Vehicular Environments, IEEE Unapproved Draft Std. P802.11p/D8.0, Jul. 2009.

[10] J. Mo, H.-S. W. So and J. Walrand, "Comparison of Multichannel MAC Protocols," IEEE Trans. Mobile Computing, vol. 7, no. 1, pp. 50-65, 2008.

[11] L. Le, "Performance Analysis of Multi-Channel MAC Protocols in Multi-Hop Ad Hoc Networks," GLOBECOM 2010, pp. 1 -6, Dec. 2010.

[12] Q. Wang, S. Leng, H. Fu, Y. Zhang and H. Weerasinghe, "An Enhanced Multi-Channel MAC for the IEEE 1609.4 Based Vehicular Ad Hoc Networks," Proc. INFOCOM IEEE Conf. Computer Communications Workshops, pp. 1-2, 2010.

[13] N. Lu, Y. Ji, F. Liu and X. Wang, "A Dedicated Multi-Channel MAC Protocol Design for VANET with Adaptive Broadcasting," Proc. IEEE Wireless Communications and Networking Conf. (WCNC), pp. 1-6, 2010.

[14] N. Lu, X. Wang, P. Wang, P. Lai and F. Liu, "A distributed reliable multi-channel MAC protocol for vehicular ad hoc networks," Proc. IEEE Intelligent Vehicles Symp, pp. 1078-1082, 2009.

[15] J. So and N. H. Vaidya, "Multi-channel mac for ad hoc networks: handling multi-channel hidden terminals using a single transceiver," Proc. 5th ACM international symposium on Mobile ad hoc networking and computing, ACM, pp. 222-233, 2004.

[16] A. Tzamaloukas and J. J. Garcia-Luna-Aceves, "Channel-hopping multiple access," Proc. ICC 2000, vol. 1, pp. 415-419, 2000.

[17] A. Tzamaloukas and J. J. Garcia-Luna-Aceves, "Channel-Hopping Multiple Access with Packet Trains for Ad Hoc Networks,"Proc. MoMuC 00, Oct. 2000.

[18] P. Bahl and R. Chandra, "SSCH: Slotted Seeded Channel Hopping for Capacity Improvement in IEEE 802.11 Ad-Hoc Wireless Networks," Proc. ACM Mobicom, pp. 216-230, Sep. 2004.

[19] Hoi-Sheung, W. So, J. Walrand and J. Mo, "McMAC: A Parallel Rendezvous Multi-Channel MAC Protocol,"WCNC 2007, pp. $334-339$, Mar. 2007.

[20] T. Luo, M. Motani and V. Srinivasan, "CAM-MAC: A Cooperative Asynchronous Multi-Channel MAC Protocol for Ad Hoc Networks," Proc. 3rd Int. Conf. Broadband Communications, Networks and Systems BROADNETS 2006, pp. 1-10, 2006.

[21] J. Lee, J. Mo, T. M. Trung, J. Walrand and H.-S. W. So, "Design and Analysis of a Cooperative Multichannel MAC Protocol for Heterogeneous Networks," IEEE Trans. Vehicular Technology, vol. 59, no. 7, pp. 3536 -3548, Sep. 2010.

[22] J. Shi, T. Salonidis and E. W. Knightly, "Starvation mitigation through multi-channel coordination in CSMA multi-hop wireless networks," Proc. the 7th ACM international symposium on Mobile ad hoc networking and computing, ACM, pp. 214-225, 2006.

[23] [Online]. Available: http://www.isi.edu/nsnam/ns/ 